\title{
Robust Cooperative Guidance Law for Simultaneous Arrival
}

DOI:

10.1109/TCST.2018.2804348

\section{Document Version}

Accepted author manuscript

Link to publication record in Manchester Research Explorer

\section{Citation for published version (APA):}

Li, Z., \& Ding, Z. (2018). Robust Cooperative Guidance Law for Simultaneous Arrival. IEEE Transactions on Control Systems Technology. https://doi.org/10.1109/TCST.2018.2804348

\section{Published in:}

IEEE Transactions on Control Systems Technology

\section{Citing this paper}

Please note that where the full-text provided on Manchester Research Explorer is the Author Accepted Manuscript or Proof version this may differ from the final Published version. If citing, it is advised that you check and use the publisher's definitive version.

\section{General rights}

Copyright and moral rights for the publications made accessible in the Research Explorer are retained by the authors and/or other copyright owners and it is a condition of accessing publications that users recognise and abide by the legal requirements associated with these rights.

\section{Takedown policy}

If you believe that this document breaches copyright please refer to the University of Manchester's Takedown Procedures [http://man.ac.uk/04Y6Bo] or contact uml.scholarlycommunications@manchester.ac.uk providing relevant details, so we can investigate your claim.

\section{OPEN ACCESS}




\title{
Robust Cooperative Guidance Law for Simultaneous Arrival
}

\author{
Zhenhong Li, Zhengtao Ding, Senior Member, IEEE
}

\begin{abstract}
In the cooperative simultaneous arrival problem, a group of interceptors are guided to simultaneously engage a stationary target. However, some interceptors may not follow the prescribed guidance law during the engagement, which can lead to interception failures. This brief investigates a new robust cooperative simultaneous arrival problem in the presence of misbehaving interceptors. A robust cooperative guidance law (RCGL) integrated with a local filtering algorithm is designed. Without the knowledge of faulty interceptors (no fault diagnosis procedure is needed), the RCGL achieves a simultaneous arrival between normal interceptors if the misbehavior of faulty interceptors can be characterized by a threat model. By characterizing the contracting behavior of the maximum gap between impact time estimates of normal interceptors, sufficient conditions are established to guarantee the convergence of RCGL. Furthermore, regardless the network connections, the impact times of normal interceptors are upper bounded by the maximum initial time-togo estimate of normal interceptors. Numerical comparison studies demonstrate the guidance performance of RCGL.
\end{abstract}

Index Terms-Robust cooperative guidance law; cooperative control; simultaneous arrival.

\section{INTRODUCTION}

The simultaneous arrival problem of multiple interceptors has become more interesting over the past few years (see, e.g., [1]-[8]). In general, the problem can be solved by two methods: 1) individual homing, e.g., [1]-[3]; 2) cooperative homing, e.g., [4]-[8]. Compared to the individual homing, the cooperative homing requires no predetermination of a common impact time. The group of interceptors synchronize the impact time by addressing the consensus problem of timeto-go estimates of interceptors.

The advancement of defense systems poses new challenges in homing guidance. It is important to increase the reliability of the cooperative guidance, especially, when some interceptors are destroyed or disturbed by the defense system of the target. However, studies on the robust simultaneous arrival problem in the presence of misbehaving interceptors are rare. The authors in [6] and [7] proposed two finite-time cooperative guidance laws (FTCGLs) based on classic graph theory and discussed the guidance performances of FTCGLs under communication faults and actuator faults. But the faulty interceptor must remain controllable and can not be the root of the communication structure. Since the defense systems of the target may destroy or disturb the interceptor and selffaults may happen during the engagement, the controllability

Z. Li and Z. Ding are with School of Electrical and Electronic Engineering, University of Manchester, Sackville Street Building, Manchester M13 9PL, UK (emails: zhenhong.li@postgrad.manchester.ac.uk; zhengtao.ding@manchester.ac.uk). of faulty interceptors is hard to preserve. It remains an open problem to design a robust guidance law such that 1) all normal interceptors can reach the target without identifying faulty interceptors; 2) all normal interceptors reach the target at the same time as much as possible.

In this brief, we consider a new robust cooperative simultaneous arrival problem when some interceptors may not follow the prescribed guidance law during the engagement. The unknown dynamics caused by faulty interceptors make the cooperative guidance design for the normal interceptors difficult. Inspired by the time-to-go approximate model in [4] and the notion of network robustness [9]-[11], we integrate a local filtering algorithm with other cooperative guidance law and present a useful robust cooperative guidance law (RCGL). If the misbehavior of faulty interceptors can be characterized by a threat model (each faulty interceptor sends the same value to all of its out-neighbors at each time-step), the RCGL can reduce the variance of impact times between normal interceptors without identifying faulty interceptors. Regardless the network connections, the impact times of normal interceptors are upper bounded by the maximum initial time-to-go estimate of normal interceptors, which can be seen as a safety condition. By discarding some extreme time-to-go estimates of in-neighbors at each time-step, the integrated local filtering algorithm of RCGL filters undesirable dynamics caused by faulty interceptors. Sufficient conditions are established to guarantee the consensus of time-to-go estimates of normal interceptors. The convergence analysis of RCGL is based on characterizing the contracting behavior of the maximum gap between impact time estimates of normal interceptors. Numerical comparison results demonstrate the effective guidance performance of RCGL.

The remainder of this brief is organized as follows. Section II formulates the robust simultaneous arrival problem with a single target and introduces the preliminaries. The main results of RCGL are given in Sections III and IV. In Section V, comparison simulation results of 5 to 1 robust engagement scenario are presented.

\section{Problem Statement}

Consider the scenario that a group of $N$ interceptors attack a stationary target on a two-dimensional plane by assuming that the lateral and longitudinal planes are decoupled by means of roll control [12]. The planar engagement geometry is shown in Fig. 1.

In Fig. 1, for the $i$ th interceptor, $r_{i}$ is the rang-to-go; $\lambda_{i}$ is the LOS angle; $\gamma_{i}$ is the flight-path angle; $\sigma_{i}$ is the 


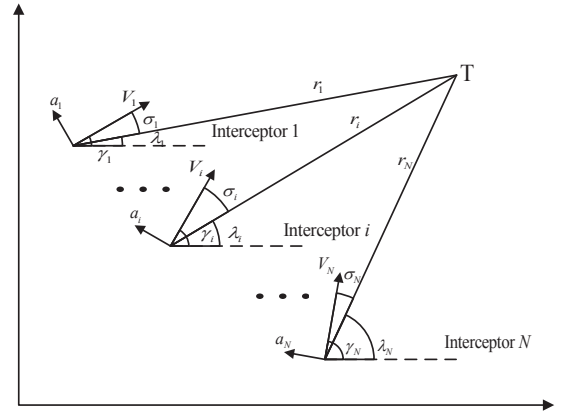

Fig. 1. Guidance geometry on $N$ to 1 engagement scenario.

heading error; $V_{i}$ is the interceptor speed, which is assumed to be constant during the engagement; $a_{i}$ is the acceleration, which is perpendicular to $V_{i}$. The planar interceptor-target engagement kinematics are given as

$$
\begin{aligned}
\dot{r_{i}} & =-V_{i} \cos \left(\sigma_{i}\right), \\
\dot{\lambda_{i}} & =-\frac{V_{i} \sin \left(\sigma_{i}\right)}{r_{i}}, \\
\dot{\gamma_{i}} & =\frac{a_{i}}{V_{i}}, \\
\sigma_{i} & =\gamma_{i}-\lambda_{i}, \quad i=1, \cdots, N .
\end{aligned}
$$

where $a_{i}$ is the control input for $i$ th interceptor.

Suppose each interceptor uses well-known proportional navigation $(\mathrm{PN})$ for homing as

$$
a_{i}=N_{s} V_{i} \dot{\lambda}_{i}
$$

where $N_{s}$ denotes the fixed navigation constant (in practice, $N_{s}$ is usually chosen as $3 \leq N_{s} \leq 5$ ). When the heading error $\sigma_{i}$ is small, the time-to-go of the $i$ th interceptor can be approximated as [4]

$$
\hat{t}_{g o, i}=\frac{r_{i}}{V_{i}}\left(\frac{\sigma_{i}^{2}}{2 N_{s}-1}+1\right), \quad i=1, \cdots, N .
$$

Note that $\sigma_{i}$ is small in general cases.

Now consider a robust simultaneous arrival problem with $N$ interceptors shown in Fig.2. The $N$ interceptors are partitioned into a set of normal interceptors $\mathcal{N}=\{i \in 1, \cdots, N$ : $i$ th interceptors is normal $\}$, and a set of faulty interceptors $\mathcal{F}=\{i \in 1, \cdots, N: i$ th interceptor is misbehaving $\}$, the number of faulty interceptor is upper bounded by $F$. The communications between interceptors happen at times $t_{0}, t_{1}, \cdots, t_{k}, \cdots$, and the communication period is $\tau$, i.e., $t_{k}-t_{k-1}=\tau$.

The communication topology among interceptors is described by the directed graph $\mathcal{G}$. The set of interceptors is defined as $\mathcal{V}=\{1, \cdots, N\}$. The adjacency matrix is defined as $\mathcal{A}=\left[\alpha_{i j}\right] \in \mathbb{R}^{N \times N}$, where $\alpha_{i i}=0$ and $\alpha_{i j}=1$ if the $i$ th interceptor can get the information from the $j$ th interceptor, otherwise $\alpha_{i j}=0$. The $j$ th interceptor is called an in-neighbor of $i$ th interceptor if $\alpha_{i j}=1$. The in-neighbors of $i$ the interceptor are defined as a set $\mathcal{V}_{i}=\left\{j \in \mathcal{V}: \alpha_{i j}=1\right\}$. The $j$ th interceptor is called an out-neighbor of $i$ th interceptor if $\alpha_{j i}=1$ (i.e., the $j$ th interceptor can get the information from the $i$ th interceptor).

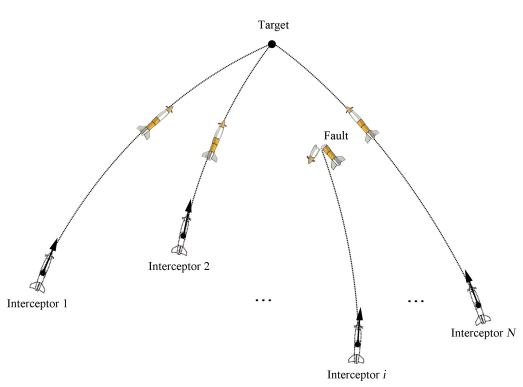

Fig. 2. $N$ to 1 robust simultaneous arrival scenario.

We make following assumptions throughout this brief.

Assumption 1: The speed of each interceptor is constant but may not be the same as that of other interceptors.

Assumption 2: Each faulty interceptor sends the same value to all of its out-neighbors at each time-step (e.g., for a faulty interceptor $i$, all the out-neighbors of $i$ receive the same value from $i$ at $t_{k}$ ).

Remark 1: In practice, Assumption 2 is nonrestrictive and easy to satisfy. For example, if the communication is realized through wireless broadcast, the faulty interceptor $i$ naturally sends the same value to all of its out-neighbors.

The objective of this brief is to design a PN based RCGL to meet following demands:

1) all normal interceptors can reach the target without the knowledge of fault interceptors.

2) all normal interceptors reach the target at the same time as much as possible.

Remark 2: Although the threat model in Assumption 2 is defined according to the communication behavior, this threat model covers a wide range of faults in practice; not only the communication faults, but also actuator faults which cause undesirable changes in $\hat{t}_{g o, i}$ are considered. It is plausible that some simple misbehaviors can be detected via an appropriate mechanism. However, for some complicate faults, especially in the short range guidance, it is hard to detect the faulty interceptors and reorganize the communications between normal interceptors. Moreover, the cooperative guidance performance will be degraded by the increasing time of fault diagnosis.

\section{RCGL With a LOCAl Filtering Algorithm}

In this section, a novel cooperative guidance law with a local filtering algorithm is designed to solve the simultaneous arrival problem. By virtue of the integrated local filtering algorithm, the proposed cooperative guidance law is robust to the misbehaviors of faulty interceptors. With exchanging the time-to-go estimates $\hat{t}_{g o, i}$ at discrete-time, the RCGL is designed as

$$
\begin{aligned}
& a_{i}(t)=N_{s}(1+k_{i} \sum_{j \in \mathcal{R}_{i}\left(t_{k}\right)} w_{i j}\left(t_{k}\right)\left(\hat{t}_{g o, i}\left(t_{k}\right)\right. \\
&\left.\left.-\hat{t}_{g o, j}\left(t_{k}\right)\right)\right) V_{i} \dot{\lambda}_{i}, \quad \forall t \in\left[t_{k}, t_{k+1}\right),
\end{aligned}
$$


where $i=1, \cdots, N, k_{i}>0$ are constants, $\mathcal{R}_{i}\left(t_{k}\right)$ is the set of retained in-neighbors of $i$ th interceptor at $t_{k}, \hat{t}_{g o, j}\left(t_{k}\right)$ and $w_{i j}\left(t_{k}\right)$ are defined in the local filtering algorithm running at times $\left\{t_{k}\right\}$.

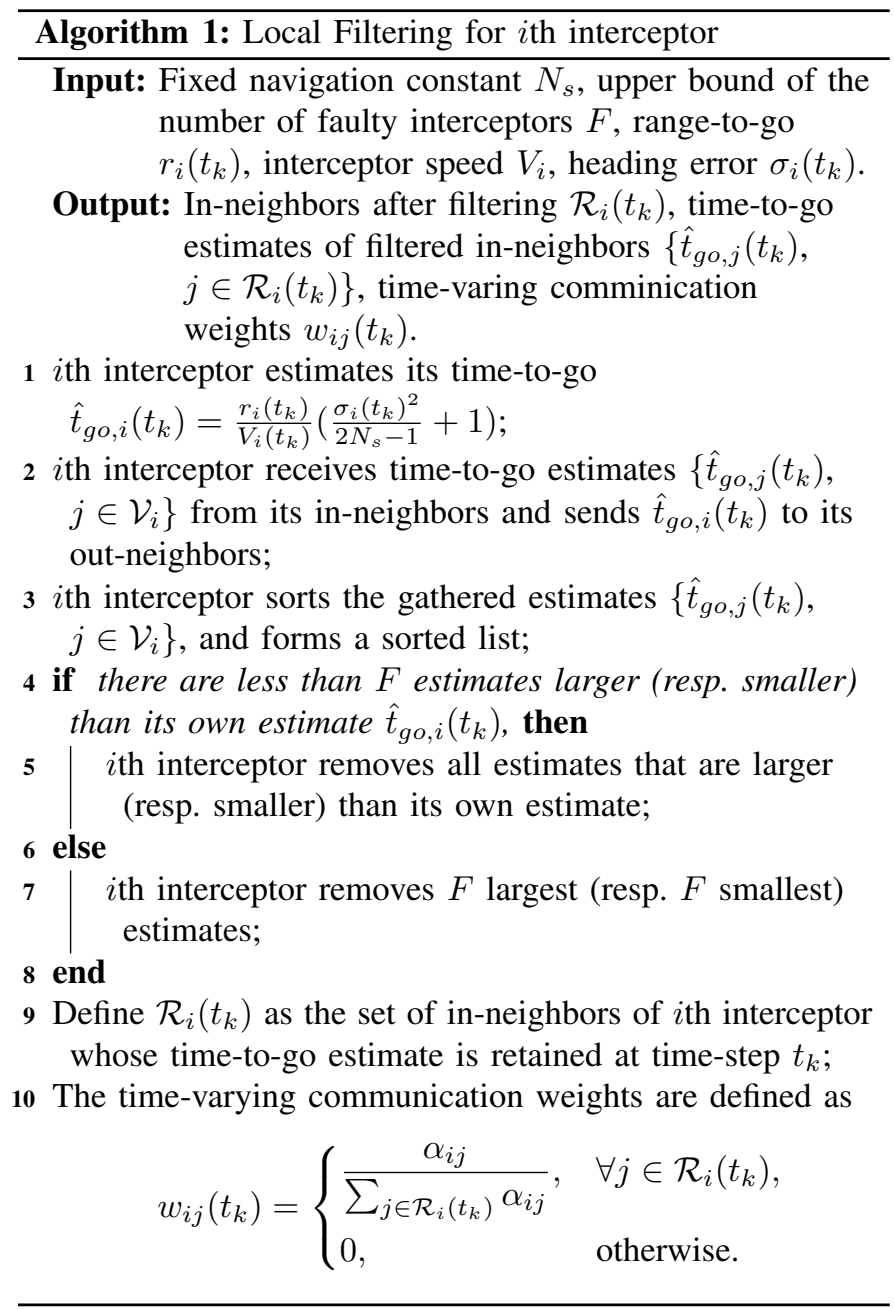

The RCGL has a simple structure which is composed of a traditional PN feedback loop, a cooperative timeto-go feedback loop and a novel local filtering algorithm. Note that RCGL is a continuous-time guidance law; however, the communications between interceptors are in discrete-time structures. The retained in-neighbors of $i$ th interceptor are switching due to the local filtering algorithm. The RCGL uses the relative time-to-go errors $\sum_{j \in \mathcal{R}_{i}\left(t_{k}\right)} w_{i j}\left(t_{k}\right)\left(\hat{t}_{g o, i}\left(t_{k}\right)-\hat{t}_{g o, j}\left(t_{k}\right)\right)$ to adjust the curvature of the interceptors's trajectories; interceptors with smaller time-to-go estimates take detours, and interceptors with larger time-to-go estimates take shortcuts. When $\sum_{j \in \mathcal{R}_{i}\left(t_{k}\right)} w_{i j}\left(t_{k}\right)\left(\hat{t}_{g o, i}\left(t_{k}\right)-\hat{t}_{g o, j}\left(t_{k}\right)\right)=0$ at any $t_{k}$, the simultaneous arrival is achieved, and RCGL becomes PN with fixed navigation constant $N_{s}$. In Algorithm 1, no additional procedure (e.g., fault detection) is needed, and the information used in the algorithm is the same as that of existing PN based cooperative guidance laws. The data flow structure of $i$ th interceptor in the cooperative guidance is shown in Fig. 3. In the figure, at each time-step, $i$ th interceptor communicates with its neighbors, removes some time-to-go estimates of in-neighbors according to the rules in Algorithm 1 and recalculates the communication weights $w_{i j}(\cdot)$. The controller calculates the acceleration command by using continuously measurements and sampled time-to-go estimates. The rigorous convergence analysis of RCGL is performed in the following section.

\section{Convergence Analysis of RCGL}

In this section, sufficient conditions are established to guarantee the convergence of RCGL. Before introducing the main results of this section, an important definition is given as follow

Definition $1((r, s)$-robust graphs): For two positive integers $r$ and $s$, a graph $\mathcal{G}$ is said to be $(r, s)$-robust if for any two disjoint nonempty subsets $\mathcal{S}_{1}, \mathcal{S}_{2} \in \mathcal{V}$, at least one of the following holds:

1) Every interceptor in $\mathcal{S}_{1}$ has at least $r$ in-neighbors outside $\mathcal{S}_{1}$.

2) Every interceptor in $\mathcal{S}_{2}$ has at least $r$ in-neighbors outside $\mathcal{S}_{2}$.

3) There are at least $s$ interceptors in $\mathcal{S}_{1} \cup \mathcal{S}_{2}$ that each interceptor has at least $r$ in-neighbors outside its respective sets.

From lines 4-7 in Algorithm 1, we know that each interceptor periodically discards some of its in-neighbors. Definition 1 aims to capture the idea that for any two disjoint nonempty subsets, there are some interceptors within those sets that each of them has enough in-neighbors outside its respective sets. This definition plays a key role in our convergence analysis.

Define $\hat{T}_{i}\left(t_{k}\right)=\hat{t}_{g o, i}\left(t_{k}\right)+k \tau$ as the impact time estimates of $i$ th interceptors at time $t_{k}$. Note that $\hat{T}_{i}\left(t_{0}\right)=\hat{t}_{g o, i}\left(t_{0}\right)$, and we have $\hat{t}_{g o, 1}\left(t_{k}\right)=\cdots=\hat{t}_{g o, N}\left(t_{k}\right)$ if $\hat{T}_{1}\left(t_{k}\right)=$ $\cdots=\hat{T}_{N}\left(t_{k}\right)$. Define $m\left(t_{k}\right)=\min \left\{\hat{T}_{i}\left(t_{k}\right), \forall i \in \mathcal{N}\right\}$ and $M\left(t_{k}\right)=\max \left\{\hat{T}_{i}\left(t_{k}\right), \forall i \in \mathcal{N}\right\} ; m\left(t_{k}\right)$ and $M\left(t_{k}\right)$ are the lower and upper bounds of the impact time estimates of normal interceptors at $t_{k}$, respectively.

Theorem 1: Suppose that there are at most $F$ faulty interceptors within a group of $N$ interceptors. Under Assumptions 1, 2 and RCGL, the impact time estimates of normal interceptors $\hat{T}_{i}\left(t_{k}\right), \forall i \in \mathcal{N}$ are bounded within $\left[m\left(t_{0}\right), M\left(t_{0}\right)\right]$, regardless of the network connections of interceptors and the misbehaviors of faulty interceptors, if $\max \left\{k_{i}, \forall i \in \mathcal{V}\right\}<\frac{2 N_{s}-1}{N_{s} \pi^{2} \tau}$.

Proof: Since $\sigma_{i}$ are small angles in general cases [4]; thus, we have $\sin \left(\sigma_{i}\right)=\sigma_{i}$ and $\cos \left(\sigma_{i}\right)=1-\frac{\sigma_{i}^{2}}{2}$. Substituting (3) into (1), we have

$$
\begin{aligned}
\dot{r}_{i}= & -V_{i}\left(1-\frac{\sigma_{i}^{2}}{2}\right), \\
\dot{\sigma}_{i}= & -\frac{V_{i} \sigma_{i}}{r_{i}}\left(N_{s}-1+N_{s} k_{i} \sum_{j \in \mathcal{R}_{i}\left(t_{k}\right)} w_{i j}\left(t_{k}\right)\right. \\
& \left.\cdot\left(\hat{t}_{g o, i}\left(t_{k}\right)-\hat{t}_{g o, j}\left(t_{k}\right)\right)\right), \quad \forall t \in\left[t_{k}, t_{k+1}\right)
\end{aligned}
$$

where $i=1, \cdots, N$. 


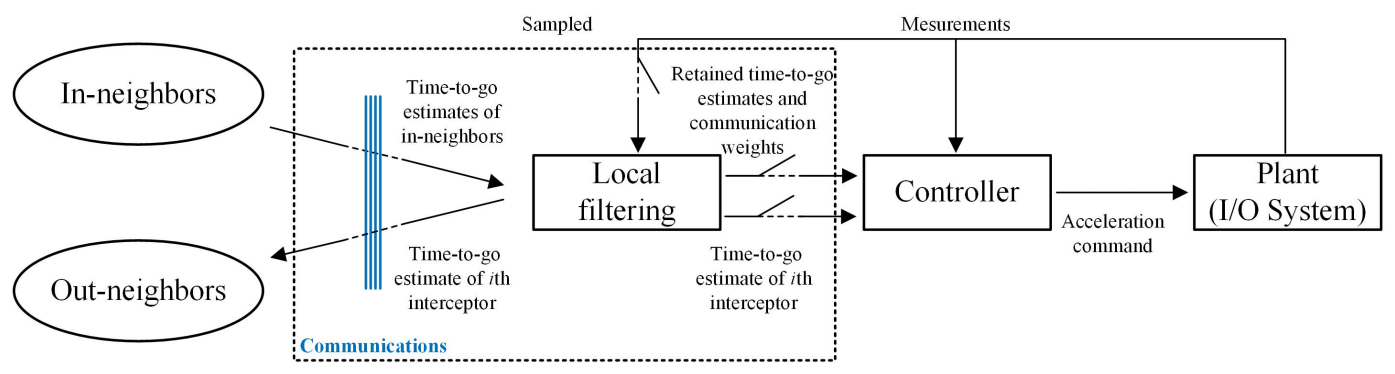

Fig. 3. Data flow structure of $i$ th interceptor under RCGL

We can get the difference equation from the above results and (2)

$$
\begin{aligned}
\hat{t}_{g o, i}\left(t_{k+1}\right)= & \hat{t}_{g o, i}\left(t_{k}\right)+\int_{0}^{\tau} \dot{\hat{t}}_{g o, i}(t) \\
= & \int_{0}^{\tau} \frac{\dot{r}_{i}}{V_{i}}\left(1+\frac{\sigma_{i}^{2}}{2\left(2 N_{s}-1\right)}\right)+\frac{r_{i} \sigma_{i} \dot{\sigma}_{i}}{V_{i}\left(2 N_{s}-1\right)} d t \\
& +\hat{t}_{g o, i}\left(t_{k}\right) \\
= & -K_{i} \sum_{j \in \mathcal{R}_{i}\left(t_{k}\right)} w_{i j}\left(t_{k}\right)\left(\hat{t}_{g o, i}\left(t_{k}\right)-\hat{t}_{g o, j}\left(t_{k}\right)\right) \\
& +\hat{t}_{g o, i}\left(t_{k}\right)-\tau,
\end{aligned}
$$

where $K_{i}=\frac{N_{s} \sigma_{i}^{2} k_{i} \tau}{2 N_{s}-1}$. Since $\max \left\{k_{i}, \forall i \in \mathcal{V}\right\}<\frac{2 N_{s}-1}{N_{s} \pi^{2} \tau}$ and $\sigma_{i}^{2} \leq \pi^{2}$, we have $0<K_{i}<1, \forall i=1, \cdots, N$.

The difference equations of impact time estimates are given as

$$
\begin{aligned}
\hat{T}_{i}\left(t_{k+1}\right)= & \left(1-K_{i} \sum_{j \in \mathcal{R}_{i}\left(t_{k}\right)} w_{i j}\left(t_{k}\right)\right) \hat{T}_{i}\left(t_{k}\right) \\
& +K_{i} \sum_{j \in \mathcal{R}_{i}\left(t_{k}\right)} w_{i j}\left(t_{k}\right) \hat{T}_{j}\left(t_{k}\right) .
\end{aligned}
$$

Note that $\sum_{j \in \mathcal{R}_{i}\left(t_{k}\right)} w_{i j}\left(t_{k}\right)=0$ if $\mathcal{R}_{i}\left(t_{k}\right)=\emptyset$. Otherwise we have that $\sum_{j \in \mathcal{R}_{i}\left(t_{k}\right)} w_{i j}\left(t_{k}\right)=1$.

For a normal interceptor $i \in \mathcal{N}$, consider following situations.

1) $\mathcal{R}_{i}\left(t_{k}\right)=\emptyset$.

2) $\mathcal{R}_{i}\left(t_{k}\right) \subseteq \mathcal{N}$.

3) $\mathcal{R}_{i}\left(t_{k}\right) \cap \mathcal{F} \neq \emptyset$, where $\emptyset$ denotes the empty set.

For the first situation, all in-neighbors of $i$ th interceptor are removed. Then the RCGL becomes a PN guidance law and $\hat{T}_{i}\left(t_{k+1}\right)=\hat{T}_{i}\left(t_{k}\right) \in\left[m\left(t_{k}\right), M\left(t_{k}\right)\right]$.

For the second situation, all the remaining interceptors are normal. Since $0<K_{i}<1$, together with (6), we can get that $T_{i}\left(t_{k+1}\right)$ is a convex combination of itself and $\hat{T}_{j}\left(t_{k}\right), \forall j \in$ $\mathcal{R}_{i}\left(t_{k}\right)$. Then, $\hat{T}_{i}\left(t_{k+1}\right) \in\left[m\left(t_{k}\right), M\left(t_{k}\right)\right]$.

For the third situation, there is at least one faulty interceptor in $\mathcal{R}_{i}\left(t_{k}\right)$. For the reason that $i$ th interceptor removes at most $F$ in-neighbors which have larger (resp. smaller) time-togo estimates than $i$ th interceptor, and the number of faulty interceptor is upper bounded by $F$, there must be at least one normal interceptor (can be $i$ th interceptor) in $\mathcal{N}$ that has a larger time-to-go estimate than the time-to-go estimates of all faulty interceptors in $\mathcal{R}_{i}\left(t_{k}\right) \cap \mathcal{F}$; furthermore, there must be at least one normal interceptor (can be $i$ th interceptor) in
$\mathcal{N}$ that has a smaller time-to-go estimate than the time-to-go estimates of all faulty interceptors in $\mathcal{R}_{i}\left(t_{k}\right) \cap \mathcal{F}$. Then we have $\hat{T}_{i}\left(t_{k+1}\right) \in\left[m\left(t_{k}\right), M\left(t_{k}\right)\right]$. Based on the above analysis, we can conclude that $\left\{M\left(t_{k}\right)\right\}$ and $\left\{m\left(t_{k}\right)\right\}$ are monotone and bounded sequences. Furthermore, $\hat{T}_{i}\left(t_{k+1}\right) \in\left[m\left(t_{0}\right), M\left(t_{0}\right)\right]$.

Remark 3: Theorem 1 shows that the impact time estimates of normal interceptors are always within $\left[\min \left\{\hat{t}_{g_{o, i}}\left(t_{0}\right), \forall i \in \mathcal{N}\right\}, \quad \max \left\{\hat{t}_{g_{o, i}}\left(t_{0}\right), \forall i \in \mathcal{N}\right\}\right] . \quad$ Note that $\hat{t}_{g o, i}=0$ only when $r_{i}(t)=0$ (i.e., $i$ th interceptor reaches the target). Together with Theorem 1, we can obtain $\hat{t}_{g o, i}\left(t_{k}\right)+k \tau \leq \max \left\{\hat{t}_{g o, i}\left(t_{0}\right), \forall i \in \mathcal{N}\right\}$, which implies that all normal interceptors will reach the target no later than $\max \left\{\hat{t}_{g_{0}, i}\left(t_{0}\right), \forall i \in \mathcal{N}\right\}$, regardless misbehaviors of faulty interceptors.

Remark 4: When the communication period $\tau$ is sufficiently small, any positive constant $k_{i}$ can guarantee the boundedness of $\hat{T}_{i}\left(t_{k}\right)$.

Theorem 2: Suppose that there are at most $F$ faulty interceptors within a group of $N$ interceptors and the communication graph $\mathcal{G}$ is $(F+1, F+1)$-robust. Under Assumptions 1 and 2, the difference between the upper and lower bounds of the impact time estimates of normal interceptors, i.e., $M\left(t_{k}\right)-m\left(t_{k}\right)$, monotonously converges to 0 if $\max \left\{k_{i}, \forall i \in \mathcal{V}\right\}<\frac{2 N_{s}-1}{N_{s} \pi^{2} \tau}$.

Proof: The proof is stated in Appendix.

Corollary 1: Under assumptions in Theorem 2, $\bar{V}\left(t_{k}\right)=$ $M\left(t_{k}\right)-m\left(t_{k}\right)$ exponentially converges to 0 as $k \rightarrow \infty$.

Proof: The proof follows similarly as in Theorem 2.

Remark 5: $\lim _{k \rightarrow \infty} M\left(t_{k}\right)-\lim _{k \rightarrow \infty} m\left(t_{k}\right)=0$ implies that $\lim _{k \rightarrow \infty} \hat{t}_{g o, i}, \forall i \in \mathcal{N}$ will reach an agreement and the simultaneous arrival will be achieved. Since the guidance time is finite in implementations, the difference $M\left(t_{k}\right)-m\left(t_{k}\right)$ will be nonzero. Nevertheless, $M\left(t_{k}\right)-m\left(t_{k}\right)$ is monotonously decreasing during the engagement and can be tuned by the parameters $k_{i}$ and $\tau$. Normally, under same faulty conditions and initial conditions, if $\tau$ is chosen to be sufficiently small, a system with larger gains $N_{s} k_{i}$ will have a smaller $M(t)-m(t)$ at the time instant $t$.

Remark 6: Under Assumption 2, the faulty interceptors are allowed to send any value as a time-to-go estimate to their outneighbors. If a fault happens during the engagement, we do not assume the faulty interceptors know the time-to-go estimates of other interceptors.

Remark 7: In implementations, $F$ is estimated according to the communication network reliability, the failure rate of interceptors and the robustness of RCGL that we want to 


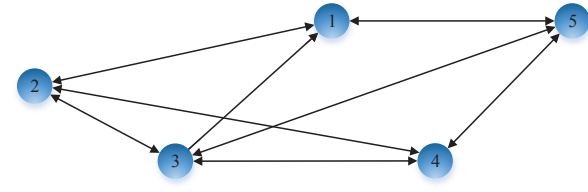

Fig. 4. Communication topology $\mathcal{G}_{1}$.

achieve. Note that we can get the maximum feasible value of $F$ if the communication network has been set up. Furthermore, there is a tradeoff between the communication load and the robustness of RGCL. In general, the RGCL with larger $F$ will have a higher communication cost. In this brief, we assume $F$ is available. Specific steps for constructing a $(r, s)$-robust graph are given in [10].

Remark 8: In light of the existing cooperative guidance laws, there are only a few results cover the simultaneous arrival under fault conditions ( [6], [7]). These results all based on the assumptions that faulty interceptors can still reach the target (i.e., the faulty interceptor is still controllable) and all the interceptors send correct time-to-go estimates to their out-neighbors. However, when faulty interceptors can not reach the target (e.g., actuator fault happens, $a_{i}=0$ at all times) or faulty interceptors keep sending incorrect time-togo estimates to their out-neighbors, the existing cooperative guidance laws can not guarantee the simultaneous arrival for normal interceptors. In fact, in the worst case, the normal interceptors which influenced by faulty interceptors can not even reach the target. Different from these existing guidance laws, by virtue of the embedded local filtering algorithm, the RCGL filters the undesirable effects of faulty interceptors and achieves the simultaneous arrival between normal interceptors.

\section{Numerical Simulation}

In this section, simulation studies are carried out to investigate the characteristics of RCGL. Consider an engagement scenario that 5 interceptors attack a single stationary target with initial conditions shown in Table I. The communication topology $\mathcal{G}_{1}$ between interceptors is shown in Fig. 4. The communication topology $\mathcal{G}_{1}$ is $(2,2)$-robust according to Definition 1. During the engagement, Interceptor 3 is destroyed by the defense system of the target at $3 \mathrm{~s}$.

\section{A. Guidance Performance Analysis for RCGL}

The parameters of robust guidance law are chosen as $\tau=0.05 \mathrm{~s}, N_{s}=3$ and $k_{1}=\cdots=k_{5}=3.5$. The simulation results performed with RCGL are shown in Fig. 5. As it is shown, when Interceptor 3 is intercepted at $3 \mathrm{~s}, a_{3}$ becomes $0 \mathrm{~m} / \mathrm{s}^{2}$ and the updating of $\hat{t}_{g o, 3}$ stops. From Figs. 5(b) and 5(c), we can see that time-to-go estimates of normal interceptors still reach an agreement after Interceptor 3 is intercepted; the RCGL achieves simultaneous arrivals by reshaping the trajectories of interceptors. In Fig. 5(d), we can see that there are some oscillations in the acceleration commands between $3 \mathrm{~s}$ and $6 \mathrm{~s}$, which are caused by the local filtering actions of RCGL. The range of acceleration commands of normal interceptors are $-92.3 \mathrm{~m} / \mathrm{s}^{2} \leq a_{i} \leq 54.3 \mathrm{~m} / \mathrm{s}^{2}$. In Fig.
6, we can observe that, before time-varying navigation gains achieve consensus, the normal interceptors with larger timeto-go estimates have larger time-varying navigation gains and the normal interceptors with smaller time-to-go estimates have smaller time-varying navigation gains. Then the time-varying navigation gains $N_{s}\left(1+k_{i} \sum_{j \in \mathcal{R}_{i}\left(t_{k}\right)} w_{i j}\left(t_{k}\right)\left(\hat{t}_{g o, i}\left(t_{k}\right)-\right.\right.$ $\left.\left.\hat{t}_{g o, j}\left(t_{k}\right)\right)\right)$ converge to a constant after $15 \mathrm{~s}$, and the RCGL becomes PN with a navigation gain $N_{s}=3$. The impact times $T_{i}$ and initial time-to-go estimates $\hat{t}_{g o, i}\left(t_{0}\right)$ are listed in the Table II. The impact times of normal interceptors are about $33.4 \mathrm{~s}$ and the dispersion of impact times is about $0.09 \mathrm{~s}$. The RCGL reduces the impact time dispersion and achieves a simultaneous arrival. Note that the impact times $T_{i}$ of normal interceptors are located within $\left[\min \left\{\hat{t}_{g o, i}\left(t_{0}\right), \forall i \in \mathcal{N}\right\}\right.$, $\left.\max \left\{\hat{t}_{g o, i}\left(t_{0}\right), \forall i \in \mathcal{N}\right\}\right]$, which can be seen as a safety condition.

\section{B. Comparison with other cooperative guidance law which has a fault diagnosis procedure}

For the sake of guidance performance comparisons, a cooperative guidance law without local filtering algorithm is chosen as

$$
a_{i}(t)=N_{s}\left(1+k_{i} \sum_{j \in \mathcal{V}_{i}} \bar{w}_{i j}\left(\hat{t}_{g o, i}\left(t_{k}\right)-\hat{t}_{g o, j}\left(t_{k}\right)\right)\right) V_{i} \dot{\lambda}_{i}
$$

where,

$$
\bar{w}_{i j}\left(t_{k}\right)= \begin{cases}\frac{\alpha_{i j}}{\sum_{j \in \mathcal{V}_{i}} \alpha_{i j}}, & \forall j \in \mathcal{V}_{i}, \\ 0, & \text { otherwise. }\end{cases}
$$

Each interceptor is integrated with a fault diagnosis procedure which can detect the faulty interceptor and reorganize the communication between normal interceptors.

All parameters are chosen to be the same as that of Section $\mathrm{V}$-A. Suppose that the fault diagnosis procedure needs $8 \mathrm{~s}$ to detect the fault and reorganize communications between normal interceptors (i.e., the fault diagnosis procedure accomplishes at $11 \mathrm{~s}$ ). The simulation results in Fig. 7 show that guidance law (7) can achieve the simultaneous arrival by adding a fault diagnosis procedure. However, the trajectories of $\hat{t}_{g o, i}$ and $a_{i}$ are strong influenced by the misbehaviors of Interceptor 3 during $3 \mathrm{~s}$ to $11 \mathrm{~s}$. In Fig. 7(b), the time-to-go estimate of Interceptor 3 becomes a leader, and all time-to-go estimates of normal interceptors try to follow the yellow line (during $3 \mathrm{~s}$ to $11 \mathrm{~s}$ ). As depicted in Figs. 7(c) and 7(d), the accumulated errors in the time-to-go estimates of normal interceptors cause high acceleration commands $a_{i}$ and hence distort engagement trajectories. The range of acceleration commands of normal interceptors are $-92.3 \mathrm{~m} / \mathrm{s}^{2} \leq a_{i} \leq 69.87 \mathrm{~m} / \mathrm{s}^{2}$. The control costs $\int_{t_{0}}^{T_{i}}\left|a_{i}\right| d t$ of two guidance laws are shown in Fig. 8. The guidance law (7) use more control efforts than RCGL, since the normal interceptors take detours before fault diagnosis procedure accomplishes. The impact times $T_{i}$ and initial time-to-go estimates $\hat{t}_{g o, i}\left(t_{0}\right)$ are listed in the Table III. Note that the impact times of interceptors are about 38.4 
TABLE I

ENGAGEMENT SCENARIO FOR 5 INTERCEPTORS

\begin{tabular}{cccccc}
\hline Parameters & Interceptor 1 & Interceptor 2 & Interceptor 3 & Interceptor 4 & Interceptor 5 \\
\hline Initial range-to-go $(\mathrm{km})$ & 7 & 7.5 & 7 & 10 & 8 \\
Initial position $(\mathrm{km})$ & $(-6.06,-3.5)$ & $(-7.05,-2.57)$ & $(-7,0)$ & $(-9.4,-3.42)$ & $(-7.88,1.39)$ \\
Initial heading error(deg) & -20 & 10 & -15 & 15 & 15 \\
Velocity(m/s) & 240 & 225 & 220 & 325 & 240 \\
Target position & $(0,0)$ & $(0,0)$ & $(0,0)$ & $(0,0)$ & $(0,0)$ \\
\hline
\end{tabular}

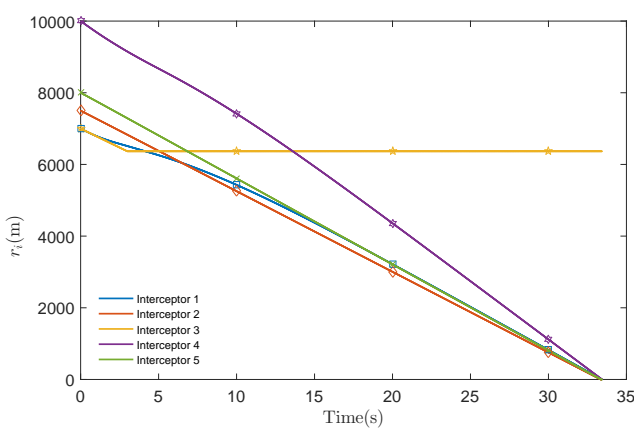

(a)

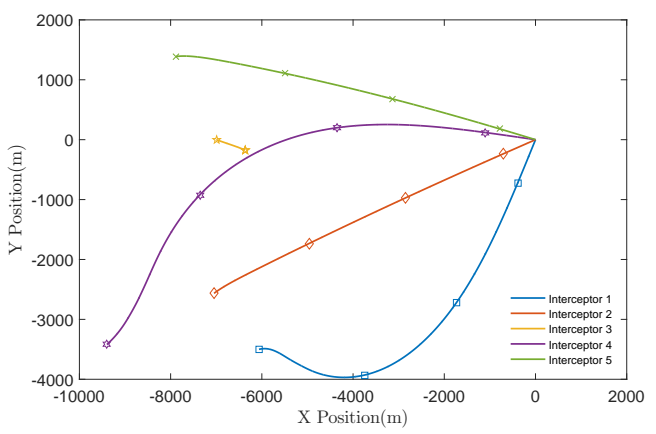

(c)

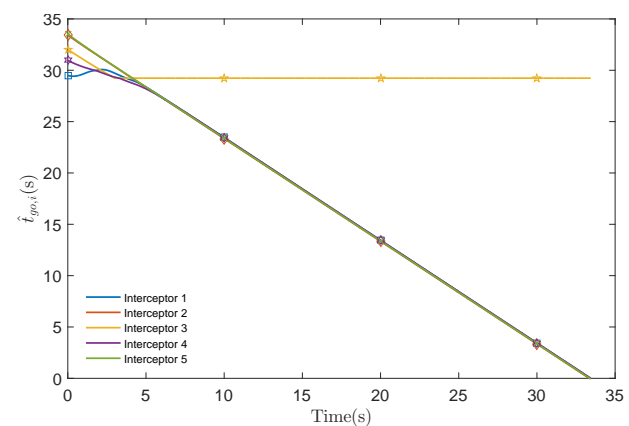

(b)

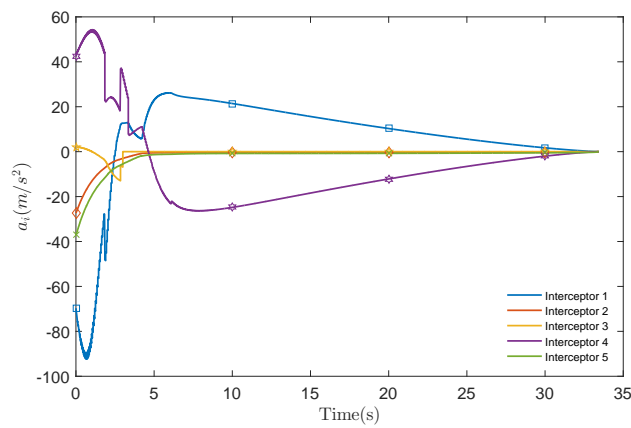

(d)

Fig. 5. Simulation results of RCGL. (a) Range-to-go $r_{i}, \forall i=1, \cdots, 5$; (b) Time-to-go estimates $\hat{t}_{g o, i}, \forall i=1, \cdots, 5$; (c) Trajectories of interceptors; (d) Acceleration commands $a_{i}, \forall i=1, \cdots, 5$.

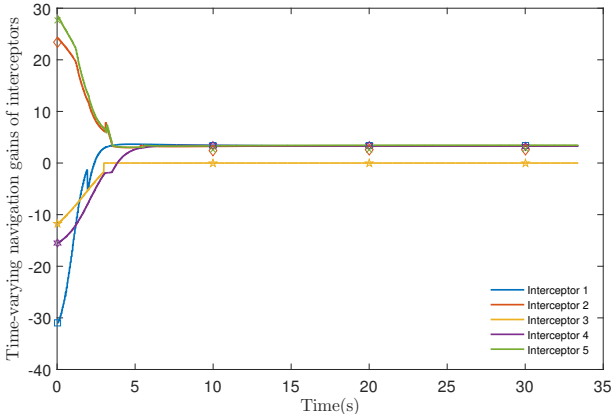

Fig. 6. Time-varying navigation gains $N_{s}\left(1+k_{i} \sum_{j \in \mathcal{R}_{i}\left(t_{k}\right)} w_{i j}\left(t_{k}\right)\right.$ $\left.\left(\hat{t}_{g o, i}\left(t_{k}\right)-\hat{t}_{g o, j}\left(t_{k}\right)\right)\right), \forall i=1, \cdots, 5$.

$\mathrm{s}$, which is larger than $\max \left\{\hat{t}_{\text {go,i }}\left(t_{0}\right), \forall i \in \mathcal{N}\right\}$. In fact, the impact time of the normal interceptor under guidance law (7) is related to the time of fault diagnosis; if the time of fault diagnosis is longer, the impact time will be delayed. If the fault diagnosis fails, the guidance law (7) can not even guarantee
TABLE II

IMPACT TIME OF NORMAL INTERCEPTORS UNDER RCGL

\begin{tabular}{ccccc}
\hline & Interceptor 1 & Interceptor 2 & Interceptor 4 & Interceptor 5 \\
\hline Initial time-to-go(s) & 29.49 & 33.43 & 30.96 & 33.54 \\
Impact time(s) & 33.42 & 33.35 & 33.44 & 33.38 \\
\hline Impact time dispersion(s) & \multicolumn{5}{c}{0.09} \\
\hline
\end{tabular}

normal interceptors reaching the target.

The above simulation results demonstrate that RCGL has better performances when faults happen during the engagement. The simultaneous arrival can be achieved without any additional fault diagnosis procedure, which enhances the reliability of the cooperative guidance.

\section{CONCLUSIONS}

This brief considers a new robust cooperative simultaneous arrival problem. A distributed cooperative guidance law RCGL is proposed based on discrete-time communications. By virtue of a novel local filtering algorithm, the RCGL can achieve a simultaneous arrival between normal interceptors without the 


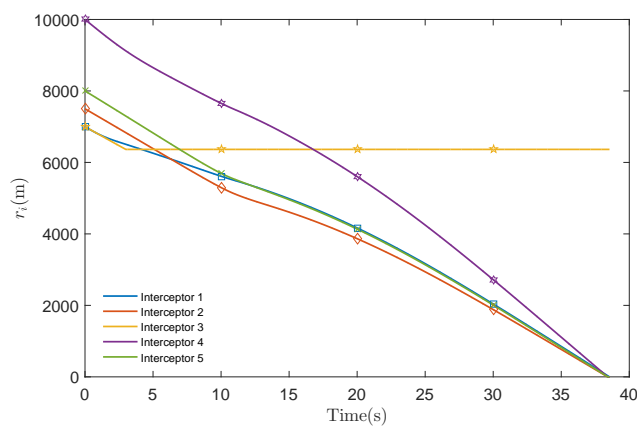

(a)

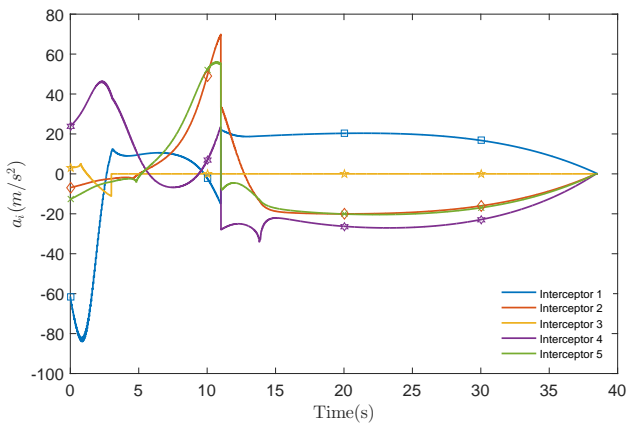

(c)

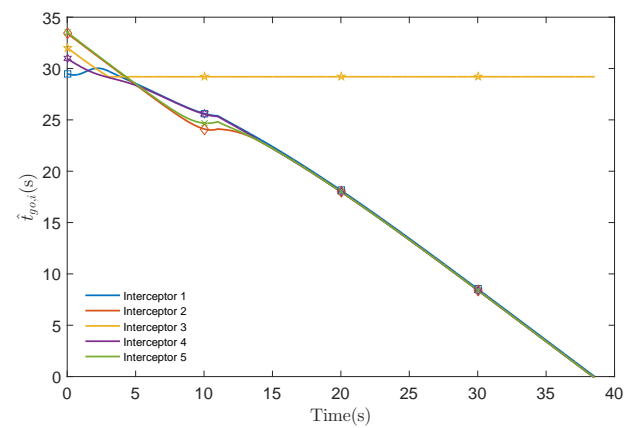

(b)

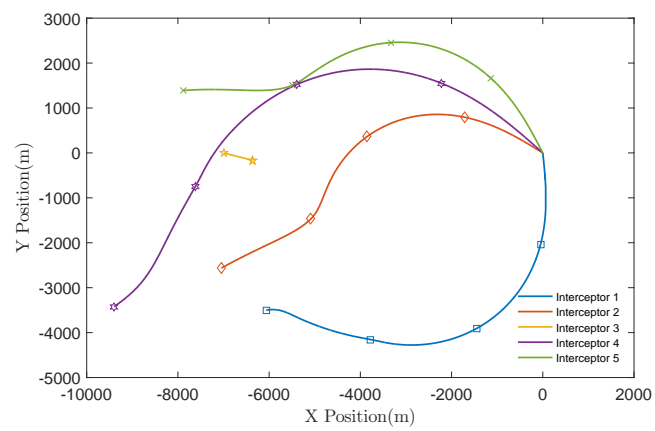

(d)

Fig. 7. Simulation results of cooperative guidance law (7) with a fault diagnosis procedure. (a) Range-to-go $r_{i}$, $\forall i=1, \cdots, 5$; (b) Time-to-go estimates $\hat{t}_{g o, i}, \forall i=1, \cdots, 5$; (c) Acceleration commands $a_{i}, \forall i=1, \cdots, 5$; (d) Trajectories of interceptors.

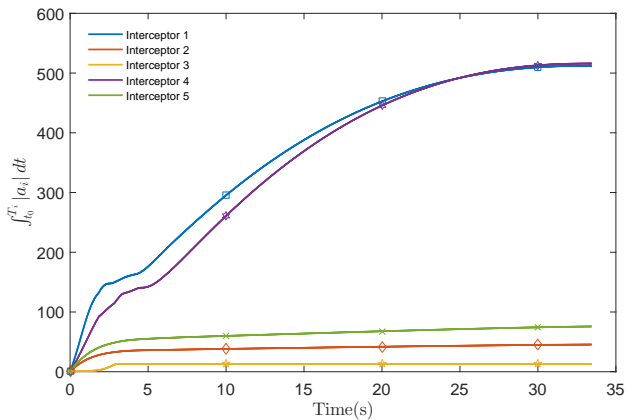

(a)

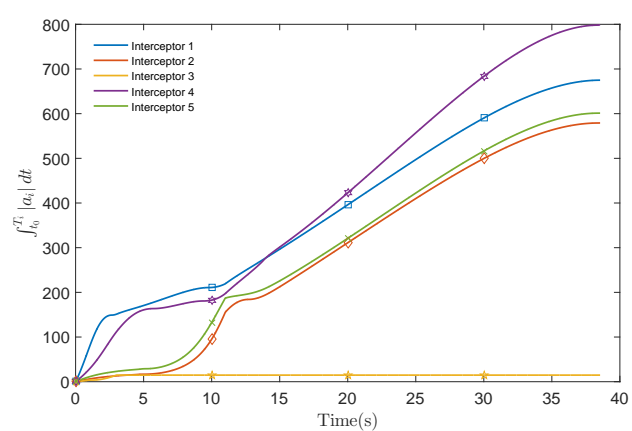

(b)

Fig. 8. Control cost of two guidance laws. (a) RCGL; (b) Cooperative guidance law (7) with fault detection procedure.

TABLE III

IMPACT TIME OF NORMAL INTERCEPTORS UNDER GUIDANCE LAW (7) WITH A FAULT DIAGNOSIS PROCEDURE

\begin{tabular}{ccccc}
\hline & Interceptor 1 & Interceptor 2 & Interceptor 4 & Interceptor 5 \\
\hline Initial time-to-go(s) & 29.49 & 33.43 & 30.96 & 33.54 \\
Impact time(s) & 38.54 & 38.41 & 38.42 & 38.42 \\
\hline Impact time dispersion(s) & \multicolumn{3}{c}{0.13} \\
\hline
\end{tabular}

knowledge of faulty interceptors (or any fault diagnosis procedure). Furthermore, the impact times of normal interceptors are upper bounded by the maximum initial time-to-go estimate of normal interceptors, regardless the network connections. Compared to the existing cooperative guidance laws, RCGL is fully distributed and requires no additional information; thus it reduces the communication burden in practice implementations. The comparison of simulation results shows that the RCGL can enhance the reliability of the cooperative guidance. Future research may include extensions to the case with manoeuvrable targets.

\section{APPENDIX \\ PROOF OF THEOREM 2}

Theorem 1 shows that $\left\{M\left(t_{k}\right)\right\}$ is nonincreasing and $\left\{m\left(t_{k}\right)\right\}$ is nondecreasing, respectively. Since every bounded monotone sequence of real numbers has a limit, $\lim _{k \rightarrow \infty} M\left(t_{k}\right)$ and $\lim _{k \rightarrow \infty} m\left(t_{k}\right)$ exist. Define $\lim _{k \rightarrow \infty} M\left(t_{k}\right)=A_{M}$ and $\lim _{k \rightarrow \infty} m\left(t_{k}\right)=A_{m}$, we will show $A_{M}=A_{m}$ by seeking a contradiction.

Suppose that $A_{M} \neq A_{m}$, we can define $\epsilon_{0}=\frac{A_{M}-A_{m}}{2}$. For any $\epsilon_{i} \in \mathcal{R}$, we define $\mathcal{T}_{M}\left(t_{k}, \epsilon_{i}\right)=\left\{i \in \mathcal{V}: \hat{T}_{i}\left(t_{k}\right)>\right.$ $\left.A_{M}-\epsilon_{i}\right\}$ and $\mathcal{T}_{m}\left(t_{k}, \epsilon_{i}\right)=\left\{i \in \mathcal{V}: \hat{T}_{i}\left(t_{k}\right)<A_{m}+\epsilon_{i}\right\}$. Note that $\mathcal{T}_{M}\left(t_{k}, \epsilon_{i}\right)$ contains normal and faulty interceptors that 
have $\hat{T}_{i}\left(t_{k}\right)$ larger than $A_{M}-\epsilon_{i}, \mathcal{T}_{m}\left(t_{k}, \epsilon_{i}\right)$ contains normal and faulty interceptors that have $\hat{T}_{i}\left(t_{k}\right)$ smaller than $A_{m}+\epsilon_{i}$.

Define a positive constant $\epsilon<\min \left\{\epsilon_{0}, \frac{\alpha^{N}}{1-\alpha^{N}} \epsilon_{0}\right\}$, where $\alpha \in(0,1)$ is a constant to be defined latter. Define $t_{k_{\epsilon}}$ as the time-step such that $M\left(t_{k_{\epsilon}}\right)<A_{M}+\epsilon$ and $m\left(t_{k_{\epsilon}}\right)>A_{m}-\epsilon$, $\forall t_{k}>t_{k_{\epsilon}}$; the existence of $t_{k_{\epsilon}}$ is guaranteed by the convergence of $\left\{M\left(t_{k}\right)\right\}$ and $\left\{m\left(t_{k}\right)\right\}$. It is obvious that $\mathcal{T}_{M}\left(t_{k_{\epsilon}}, \epsilon_{0}\right)$ and $\mathcal{T}_{m}\left(t_{k_{\epsilon}}, \epsilon_{0}\right)$ are nonempty and $\mathcal{T}_{M}\left(t_{k_{\epsilon}}, \epsilon_{0}\right) \cap \mathcal{T}_{m}\left(t_{k_{\epsilon}}, \epsilon_{0}\right)=$ $\emptyset$. Note that communication graph $\mathcal{G}$ is $(F+1, F+1)$ robust, and the number of faulty interceptors is upper bounded by $F$; if there are normal interceptors in $\mathcal{T}_{M}\left(t_{k_{\epsilon}}, \epsilon_{0}\right)$ and $\mathcal{T}_{m}\left(t_{k_{\epsilon}}, \epsilon_{0}\right)$ (i.e., $\mathcal{N} \cap \mathcal{T}_{M}\left(t_{k_{\epsilon}}, \epsilon_{0}\right) \neq \emptyset$ and $\mathcal{N} \cap \mathcal{T}_{m}\left(t_{k_{\epsilon}}, \epsilon_{0}\right) \neq \emptyset$ ), there is at least one of these normal interceptors that has at least $F+1$ inneighbors outside of its set. Note that $\mathcal{N} \cap \mathcal{T}_{M}\left(t_{k_{\epsilon}}, \epsilon_{0}\right) \neq \emptyset$ and $\mathcal{N} \cap \mathcal{T}_{m}\left(t_{k_{\epsilon}}, \epsilon_{0}\right) \neq \emptyset$ are true. Otherwise, we have $\hat{t}_{g o, i}\left(t_{k_{\epsilon}}\right) \geq$ $\frac{A_{M}+A_{m}}{2}, \forall i \in \mathcal{N}$ or $\hat{t}_{g o, i}\left(t_{k_{\epsilon}}\right) \leq \frac{A_{M}+A_{m}}{2}, \forall i \in \mathcal{N}$, which contradicts the fact that $\left\{M\left(t_{k}\right)\right\}$ and $\left\{m\left(t_{k}\right)\right\}$ monotonously converge to $A_{M}$ and $A_{m}$ respectively. Without loss of generality, suppose that the normal interceptor $i \in \mathcal{T}_{M}\left(t_{k_{\epsilon}}, \epsilon_{0}\right) \cap \mathcal{N}$ has at least $F+1$ in-neighbors outside of $\mathcal{T}_{M}\left(t_{k_{\epsilon}}, \epsilon_{0}\right)$. Then, we have $\hat{T}_{j}\left(t_{\left.k_{\epsilon}\right)} \leq A_{M}-\epsilon_{0}, \forall j \in \mathcal{V}_{i} \cap \complement_{\mathcal{V}} \mathcal{T}_{M}\left(t_{k_{\epsilon}}, \epsilon_{0}\right)\right.$, where $\complement_{\mathcal{V}} \mathcal{T}_{M}\left(t_{k_{\epsilon}}, \epsilon_{0}\right)$ denotes the relative complement of $\mathcal{T}_{M}\left(t_{k_{\epsilon}}, \epsilon_{0}\right)$ with respect to $\mathcal{V}$. Since normal interceptor $i$ removes at most $F$ in-neighbors which are in $\complement_{\mathcal{V}} \mathcal{T}_{M}\left(t_{k_{\epsilon}}, \epsilon_{0}\right)$, at least one interceptor belongs to $\mathcal{V}_{i} \cap \complement_{\mathcal{V}} \mathcal{T}_{M}\left(t_{k_{\epsilon}}, \epsilon_{0}\right)$ will be retained in $\mathcal{R}_{i}\left(t_{k_{\epsilon}}\right)$. Assume that none of $\sigma_{i}, \forall i \in \mathcal{N}$ reaches zero before normal interceptors reach the target (i.e, there exists a positive constant $K_{m}$, such that $K_{i}>K_{m}$ ). Together with (6), we have that the impact time estimate of the normal interceptor $i$ at time $t_{k_{\epsilon}+1}$ has the following property

$$
\begin{aligned}
\hat{T}_{i}\left(t_{k_{\epsilon}+1}\right) & \leq\left(1-\frac{K_{m}}{N}\right) M\left(t_{k_{\epsilon}+1}\right)+\frac{K_{m}}{N}\left(A_{M}-\epsilon_{0}\right) \\
& \leq A_{M}-\alpha \epsilon_{0}+(1-\alpha) \epsilon \leq A_{M}-\epsilon_{1},
\end{aligned}
$$

where $\epsilon_{1}=\alpha \epsilon_{0}-(1-\alpha) \epsilon$ and $\alpha=\frac{K_{m}}{N}$. Since $0<K_{m}<$ $K_{i}<1$, we have $\alpha \in(0,1)$ and $\epsilon_{1}<\epsilon_{0}$. To get (8), we have used the fact that $w_{i j}(\cdot) \geq \frac{1}{N}$. Note that for any normal interceptor $j \notin \mathcal{T}_{M}\left(t_{k_{\epsilon}}, \epsilon_{0}\right)$, we still have $\hat{T}_{j}\left(t_{k_{\epsilon}+1}\right) \leq A_{M}-$ $\epsilon_{1}$; since such a interceptor $j$ will use its own impact time estimate $\hat{T}_{j}\left(t_{k_{\epsilon}}\right)$ at $t_{k_{\epsilon}+1}$. Similarly, if a normal interceptor $p \in \mathcal{T}_{m}\left(t_{k_{\epsilon}}, \epsilon_{0}\right)$ has at least $F+1$ in-neighbors outside of $\mathcal{T}_{m}\left(t_{k_{\epsilon}}, \epsilon_{0}\right)$, we can obtain

$$
\hat{T}_{p}\left(t_{k_{\epsilon}+1}\right) \geq A_{m}+\alpha \epsilon_{0}-(1-\alpha) \epsilon \geq A_{m}+\epsilon_{1} .
$$

Furthermore, for any normal interceptor $q \notin \mathcal{T}_{m}\left(t_{k_{\epsilon}}, \epsilon_{0}\right)$, we still have $\hat{T}_{q}\left(t_{k_{\epsilon}+1}\right) \geq A_{m}+\epsilon_{1}$.

Based on the above analysis, we know that at least one of following statement is true if both $\mathcal{N} \cap \mathcal{T}_{M}\left(t_{k_{\epsilon}}, \epsilon_{0}\right) \neq \emptyset$ and $\mathcal{N} \cap \mathcal{T}_{m}\left(t_{k_{\epsilon}}, \epsilon_{0}\right) \neq \emptyset$ :

1) At least there is one normal interceptor $i \in \mathcal{T}_{M}\left(t_{k_{\epsilon}}, \epsilon_{0}\right)$ whose $\hat{T}_{i}\left(t_{k_{\epsilon}}\right)$ decreases to $A_{M}-\epsilon_{1}$ (or below) at $t_{k_{\epsilon}+1}$. Then, we have $\left\|\mathcal{T}_{M}\left(t_{k_{\epsilon}+1}, \epsilon_{1}\right) \cap \mathcal{N}\right\|<$ $\left\|\mathcal{T}_{M}\left(t_{k_{\epsilon}}, \epsilon_{0}\right) \cap \mathcal{N}\right\|$, where $\|\cdot\|$ denotes the cardinality of a set.

2) At least there is one normal interceptor $j \in \mathcal{T}_{m}\left(t_{k_{\epsilon}}, \epsilon_{0}\right)$ whose $\hat{T}_{j}\left(t_{k_{\epsilon}}\right)$ increases to $A_{m}+\epsilon_{1}$ (or above) at $t_{k_{\epsilon}+1}$. Then, we have $\left\|\mathcal{T}_{m}\left(t_{k_{\epsilon}+1}, \epsilon_{1}\right) \cap \mathcal{N}\right\|<$ $\left\|\mathcal{T}_{m}\left(t_{k_{\epsilon}}, \epsilon_{0}\right) \cap \mathcal{N}\right\|$.
Since $\epsilon_{1}<\epsilon_{0}$, we have $\mathcal{T}_{M}\left(t_{k_{\epsilon}+1}, \epsilon_{1}\right) \cap \mathcal{T}_{m}\left(t_{k_{\epsilon}+1}, \epsilon_{1}\right)=\emptyset$. Define $\epsilon_{j}$ recursively as $\epsilon_{j}=\alpha \epsilon_{j-1}-(1-\alpha) \epsilon, \forall j \geq 1$, one can obtain $\epsilon_{j}<\epsilon_{j-1}$ and $\mathcal{T}_{M}\left(t_{k_{\epsilon}+j}, \epsilon_{j}\right) \cap \mathcal{T}_{m}\left(t_{k_{\epsilon}+j}, \epsilon_{j}\right)=\emptyset$. If there are still normal nodes in $\mathcal{T}_{M}\left(t_{k_{\epsilon}+j}, \epsilon_{j}\right) \cup \mathcal{T}_{m}\left(t_{k_{\epsilon}+j}, \epsilon_{j}\right)$, we can repeat the above analysis at $t_{k_{\epsilon}+j+1}$, then we have either $\left\|\mathcal{T}_{M}\left(t_{k_{\epsilon}+j+1}, \epsilon_{j+1}\right) \cap \mathcal{N}\right\|<\left\|\mathcal{T}_{M}\left(t_{k_{\epsilon}+j}, \epsilon_{j}\right) \cap \mathcal{N}\right\|$, or $\left\|\mathcal{T}_{m}\left(t_{k_{\epsilon}+j+1}, \epsilon_{j+1}\right) \cap \mathcal{N}\right\|<\left\|\mathcal{T}_{m}\left(t_{k_{\epsilon}+j}, \epsilon_{j}\right) \cap \mathcal{N}\right\|$, or both. Subsequently, there exists a time-step $t_{k_{\epsilon}+\bar{k}}$ such that $\mathcal{N} \cap$ $\mathcal{T}_{M}\left(t_{k_{\epsilon}+\bar{k}}, \epsilon_{\bar{k}}\right)=\emptyset$ or $\mathcal{N} \cap \mathcal{T}_{M}\left(t_{k_{\epsilon}+\bar{k}}, \epsilon_{\bar{k}}\right)=\emptyset$. Since $\left\|\mathcal{N} \cap \mathcal{T}_{M}\left(t_{k_{\epsilon}}, \epsilon_{0}\right)\right\|+\left\|\mathcal{N} \cap \mathcal{T}_{m}\left(t_{k_{\epsilon}}, \epsilon_{0}\right)\right\| \leq N$, we obtain $\bar{K}<N$. For the case $\mathcal{N} \cap \mathcal{T}_{M}\left(t_{k_{\epsilon}+\bar{k}}, \epsilon_{\bar{k}}\right)=\emptyset$, we can obtain that the impact time estimates of all normal interceptors satisfy $\hat{T}_{i}\left(t_{k_{\epsilon}+\bar{k}}\right) \leq A_{M}-\epsilon_{\bar{k}}, \forall i \in \mathcal{N}$; for the case $\mathcal{N} \cap \mathcal{T}_{m}\left(t_{k_{\epsilon}+\bar{k}}, \epsilon_{\bar{k}}\right)=\emptyset$, we can obtain that the impact time estimates of all normal interceptors satisfy $\hat{T}_{i}\left(t_{k_{\epsilon}+\bar{k}}\right) \geq A_{m}+\epsilon_{\bar{k}}$, $\forall i \in \mathcal{N}$.

In the following analysis, we will show that $\epsilon_{\bar{k}}>0$, which contradicts the fact that $\left\{M\left(t_{k}\right)\right\}$ monotonically converges to $A_{M}$ (in the first case) or that $\left\{m\left(t_{k}\right)\right\}$ monotonically converges to $A_{m}$ (in the second case). Note that

$$
\begin{aligned}
\epsilon_{\bar{k}} & =\alpha \epsilon_{\bar{k}-1}-(1-\alpha) \epsilon \\
& =\alpha^{2} \epsilon_{\bar{k}-2}-\alpha(1-\alpha) \epsilon-(1-\alpha) \epsilon \\
& =\alpha^{\bar{k}} \epsilon_{0}-\left(1-\alpha^{\bar{k}}\right) \epsilon \geq \alpha^{N} \epsilon_{0}-\left(1-\alpha^{N}\right) \epsilon .
\end{aligned}
$$

Since $\epsilon<\frac{\alpha^{N}}{1-\alpha^{N}} \epsilon_{0}$, we have $\epsilon_{\bar{k}}>0$, which provides the contradiction. Thus, we can conclude that $A_{M}=A_{m}$, the impact time estimates $\hat{T}_{i}, \forall i \in \mathcal{N}$ converge to a same value.

\section{REFERENCES}

[1] I.-S. Jeon, J.-I. Lee, and M.-J. Tahk, "Impact-time-control guidance law for anti-ship missiles," IEEE Trans. Control Syst. Technol., vol. 14, no. 2 , pp. 260-266, March 2006.

[2] G. A. Harrison, "Hybrid guidance law for approach angle and time-ofarrival control," J. Guidance, Control, Dyn., vol. 35, no. 4, pp. 1104 1114, 2012.

[3] J. I. Lee, I. S. Jeon, and M. J. Tahk, "Guidance law to control impact time and angle," IEEE Trans. Aerosp. Electron. Syst., vol. 43, no. 1, pp. 301-310, January 2007.

[4] I.-S. Jeon, J.-I. Lee, and M.-J. Tahk, "Homing guidance law for cooperative attack of multiple missiles," J. Guidance, Control, Dyn., vol. 33, no. 1, pp. 275-280, 2010.

[5] Y. Zhang, D. Yu, Y. an, and Y. Wu, "An impact-time-control guidance law for multi-missiles," in Proc. IEEE Int. Conf. Intell. Comput. Intell. Syst., vol. 2, Nov 2009, pp. 430-434.

[6] P. Zhang, H. H. Liu, X. Li, and Y. Yao, "Fault tolerance of cooperative interception using multiple flight vehicles," J. Franklin Inst., vol. 350, no. 9 , pp. $2373-2395,2013$.

[7] J. Zhou and J. Yang, "Distributed guidance law design for cooperative simultaneous attacks with multiple missiles," J. Guidance, Control, Dyn., pp. 2439-2447, 2016.

[8] M. Snyder, C. Li, and Z. Qu, "A new parameterized guidance law for cooperative air defense," in Proc. 36th AIAA Aerosp. Sci. Meeting New Horizons Forum Aerosp. Expo., 2012, p. 850.

[9] S. M. Dibaji and H. Ishii, "Resilient consensus of second-order agent networks: Asynchronous update rules with delays," Automatica, vol. 81, pp. 123 - 132, 2017

[10] H. J. LeBlanc, H. Zhang, X. Koutsoukos, and S. Sundaram, "Resilient asymptotic consensus in robust networks," IEEE J. Sel. Areas Commun., vol. 31, no. 4, pp. 766-781, April 2013.

[11] H. Zhang, E. Fata, and S. Sundaram, "A notion of robustness in complex networks," IEEE Trans. Control Netw. Syst., vol. 2, no. 3, pp. 310-320, Sept 2015.

[12] P. Gurfil, "Robust guidance for electro-optical missiles," IEEE Trans. Aerosp. Electron. Syst., vol. 39, no. 2, pp. 450-461, April 2003. 